\title{
A Review of Dispersion Compensation using Fiber Bragg Grating (FBG) in Optical Communication
}

\author{
Pranjaly Shukla ${ }^{1}$, Prof. Swatantra Tiwari ${ }^{2}$ \\ M Tech Scholar, Department of Electronics and Communication, RIT Rewa, India ${ }^{1}$ \\ Assistant Prof, Department of Electronics and Communication, RIT Rewa, India ${ }^{2}$
}

\begin{abstract}
In this modern era of communication optical fiber has emerged as an efficient and viable mode for communication. Though optical fiber communication lot of benefits, dispersion is that the main performance limiting issue. Dispersion severely degrades the performance of fiber. There are numerous ways for dispersion compensation. The fiber is usually used in telecommunication system due to its characteristics that include little size or dimension, low loss and low interferences from outside surroundings. There are numerous varieties of optical fiber compensators, however Fiber Bragg Grating (FBG) is often chosen as necessary parts to compensate the dispersion in optical communication system.
\end{abstract}

Keywords: Optical Transmission System, Fiber Bragg Grating (FBG), dispersion compensation, Optisystem simulator, parameters.

\section{INTRODUCTION}

Optical fiber evolution dates back to 1790's once the French Chappe brothers invented the primary "optical telegraph." that comprised of a series of lights mounted on towers wherever operators would relay a message from one tower to the next? In later centuries great strides were created in optical science. But the very plan of using glass to transmit info signal within the style of lightweight over long distance was developed by KAO and HOCKMAN in 1966.[2]

Optical communication systems use high carrier frequencies $(100 \mathrm{THz})$ within the visible or near-infrared region of the electromagnetic spectrum. They're generally known as lightweight wave systems to distinguish them from microwave systems, whose carrier frequency is often smaller by 5 orders of magnitude $(1 \mathrm{GHz})$. Fiber-optic communication systems are lightweight wave systems of transmission info from one place to a different by sending pulses of light through optical fiber. Such systems are deployed worldwide since 1980 and have so revolutionized the technology behind telecommunications. Indeed, the light wave technology, along with electronics, is believed to be a serious considers the advent of the "information age."

Like different Communication systems optical communication system conjointly faces issues like dispersion, attenuation and non-linear effects that result in deterioration in its performance. Among them dispersion affects the most and it's robust to overcome as compared to others [2].

Dispersion is that the main performance limiting considers optical fiber communication. Dispersion greatly hampers the performance of fiber communication. Owing to dispersion, broadens optical pulse as they travel in single mode fiber. Limiting the ultimate rate supported by fiber that causes spreading and overlapping of chips and degrades sys-tem performance owing to increase inter chip interference and reduced received optical power. Therefore if dispersion may be reduced then an extra performance may be obtained from fiber communication. There are lots of ways of dispersion compensation. Post Fiber Bragg Grating with chirp is one among these [4]. Once a pulse travels through a fiber owing to dispersion it becomes broadened. The dispersion is proportional to the length of the fiber. If the length is increased the width becomes bulk and also the magnitude reduces.

In fiber optic communication is transmitted pulses of light through an optical fiber, wherever the light forms an electromagnetic carrier wave that's modulated to transport data. This way the fiber optic is that the medium, and also the light pulses the message. Fiber optics may be a medium for carrying data from one purpose to another within the type of light. Unlike, the copper type of transmission, fiber optics isn't electrical in nature. A basic fiber optic system consists of a transmitting device that converts an electrical signal into a light signal, an optical fiber cable that carries the sunshine, and a receiver that accepts the light signal and converts it back into an electrical signal .Fiber bragg gratings (FBGs) are wide applied in optical Sensors and optical communications because of the promising Performances with electro-magnetic immunity, compactness, Remote sensing, easy fabrication and wavelength selectivity [3]. 


\section{LITERATURE SURVEY}

Kaushal Kumar et al. [1] "Performance Analysis of dispersion compensation using Fiber Bragg Grating (FBG) in Optical Communication", Author analyzed the dispersion compensation using Fiber Bragg Grating at totally different fiber lengths, the simulated transmission are analyzed on the fundamental of various parameters. By simulating a model of communication system and using the most appropriate settings of the system that include input power (dBm), fiber cable length $(\mathrm{km})$, FBG Length $(\mathrm{mm})$ and attenuation constant $(\mathrm{dB} / \mathrm{km})$ at cable section, four totally different parameters are investigated that are output power $(\mathrm{dbm})$, noise figure $(\mathrm{dB})$, gain $(\mathrm{dB})$ and $\mathrm{Q}-$ Factor $(\mathrm{db})$ at receiver. We'll see the help of eye diagrams in subsequent graph. All the results are analyzed using OPTISYSTEM simulation at 10 Giga bits per second $(\mathrm{Gb} / \mathrm{s})$ transmission systems. From the simulation result, it will conclude that, input power $(\mathrm{dBm})$, fiber cable length $(\mathrm{km})$, and attenuation coefficient $(\mathrm{dB} / \mathrm{km})$ at cable section are directly proportional to the noise figure. The noise figure could be alive of how much noise the amplifier adds to the signal. Whereas the output power $(\mathrm{dbm})$, gain $(\mathrm{dB})$ and Q-Factor $(\mathrm{db})$ are becoming decreased with the increasing optical length $(\mathrm{dBm})$, and Attenuation constant $(\mathrm{db} / \mathrm{km})$. The input power $(\mathrm{dBm})$ is increased and output power $(\mathrm{dbm})$, is increased, gain $(\mathrm{dB})$ and Q-Factor $(\mathrm{db})$ are decreased the opposite hand FBG Length $(\mathrm{db})$ is increased and output power $(\mathrm{dbm})$, noise figure $(\mathrm{dB})$, gain $(\mathrm{dB})$, and Q-Factor $(\mathrm{db})$ are nonlinear because of the usage of EDFA and also the gain has been compressed.

Mr. Tushar Kant Panda et al. [2] "Enhancing Transmission Performance of Fiber Optic Link Using Fiber Bragg Grating for Effective Dispersion Compensation", this paper discloses the utilization of FBG so as to reduce dispersion in optical fibers. From then on top of simulations we tend to get some optimized value for $50 \mathrm{~km}$ long distance communication. Optimized output will be achieved if the grating length is in between 7,8,9(mm), power input is almost 5,6,7(mw), amplifier gain is almost $20 \mathrm{db}$. The results are evaluated on the idea of vital parameters like BER, eye height, Q-factor that are found to be higher and satisfactory for the important time application and also the spectrum analyzer output is additionally found to be higher and satisfactory as shown within the above figure. Thence error free signal will be achieved within the vary of $50 \mathrm{~km}$. so as to attain least error, another methodology also can be introduced i.e. Polarization Mode Dispersion Compensation methodology.

Bibhu Prasad et al.[3] "Performance Analysis of Fiber Optical Communication using Fiber Bragg Grating as Dispersion Compensator", Author analyzed the dispersion compensation using Fiber Bragg Grating at completely different fiber lengths and at different fbg. The simulated gear has been analyzed on the essential of various parameters. The optical transmission system has been modeled by using Optisystem7.0 simulator as shown in Figure 5 so as to analyze totally different parameters of the system. From the simulation result, it will conclude that the fiber Bragg grating length and also the input power are directly proportional to the signal power. Once input power $(\mathrm{dBm})$ is increased then its output power $(\mathrm{dbm})$, is increased however Q-Factor $(\mathrm{db})$ is decreased. FBG Length $(\mathrm{db})$ is increased then output power $(\mathrm{dbm})$ and QFactor( $(\mathrm{db})$ are increased.

Sameer Anand et al[4] "Analysis on dispersion compensation using Post FBG with EDFA", the recent advances in Fiber Bragg grating technology currently permit the belief of a high performance, high speed optical fibers with good in line dispersion compensation. The characteristic of optical fiber is analyzed. The dispersion is computed by sending a NRZ pulses as an input. During this simulation we tend to are observing that the Q issue is 13.8681, Min BER is 3.06393e-044, Threshold is 4.4048e-005 and Eye Height is 0.000579026 . The Q issue is relatively low then Post DCF and Ideal FBG. Eye Height is relatively low then Pre, Post DCF and Ideal FBG. During this technique we will see the dispersion in $193.1 \mathrm{thz}$ is reduced from $2.16578 \mathrm{e}+008 \mathrm{ps} / \mathrm{ns}$ to $1.19494 \mathrm{e}+\mathrm{ps} / \mathrm{ns}$, noise is additionally reducing. OSNR ratio is improved. The ability of these signals is decreased this \{can be \} just one drawback and it can be overcome by using optical amplifier at output facet. For 193.2 thz OSNR Improves whereas dispersion will increase power of signal is de-crease. We can see these results aren't in our favor on for this signal whereas different signals are received with fine parameters. For $193.3 \mathrm{THz}$, dispersion is reduced from $3.59741 \mathrm{e}+007 \mathrm{ps} / \mathrm{nm}$ to $1.84029 \mathrm{e}+007 \mathrm{ps} / \mathrm{nm}$ whereas noise reduces and OSNR improves. For 193.4, dispersion is reduced from $6.33977 \mathrm{e}+007 \mathrm{ps} / \mathrm{nm}$ to $1.09275 \mathrm{e}+006 \mathrm{ps} / \mathrm{nm}$, noise is reduced and OSNR improves.

Ranjana Rao et al. [5] "Performance Analysis of Dispersion Compensation using FBG and DCF in WDM Systems: A Review", in this paper we have analyzed the eight channel WDM system at 15 Gbps for DCF and FBG. It's found that each of those 2 compensators, DCF similarly as FBG works well. However, the FBG compensator performs higher than DCF in high speed 8 channel WDM network. DCF and FBG are compared in terms of BER and Q-factor. From this we tend to discover that FBG Post compensation scheme performs higher than different compensation techniques.

B.Prasad et al.[6] "Fiber Bragg Grating as a Dispersion Compensator in an Optical Transmission System Using Optisystem Software", From the planning and simulation of optical transmission system, the system can transmit data using optical carrier from transmitter to receiver via optical fiber, the transmission system diagram Figure1 has been 
designed that consists of optical device light-weight because the source, modulator, single mode optical fiber because the channel, fiber Bragg grating (FBG) because the dispersion compensator, optical amplifier and also the image detector because the light-weight detector. The optical transmission system has been modeled by using Optisystem7.0 simulator as shown in Figure 5 so as to analyze totally different parameters of the system. From the simulation result, it will conclude that the fiber Bragg grating length and therefore the input power are directly proportional to the signal power. Whereas the noise is obtaining lower with the increasing length, on the opposite hand, the output power is decreased though the attenuation constant increased.

\section{METHOD}

A. Dispersion Compensation Techniques

The Dispersion can be compensated by using different dispersion compensation methods. Each of them having their own advantages and disadvantages:

- $\quad$ Dispersion Compensating Fiber (DCF):

In this technique we tend to use a fiber having large negative dispersion alongside a typical fiber. the number of light distributed by a traditional fiber in reduced or maybe nullified by using a dispersion compensating fiber having a really giant value of dispersion of opposite sign as compared to it of normal fiber[2].

There are primarily 3 schemes which will be used to install a dispersion compensating fiber-pre, post or symmetrical.

- $\quad$ Fiber Bragg Grating (FBG):

Fiber Bragg gratings since its introduction in 1980 are a topic of analysis with many applications. It's a reflective device composed of an optical fiber that contains a modulation of its core refractive index over a definite length. The Grating reflects light-weight propagating through the fiber once its wavelength corresponds to the modulation regularity.

Using fiber Bragg gratings for dispersion compensation may be a promising approach as a result of they're passive optical element fiber compatible, having low insertion losses and prices. They are used as sensors, as wavelength stabilizers for pump lasers, in narrow band WDM add drop filters and additionally as filters for dispersion compensation. Benefits of FBG are that it helps in minimizing the value of the fiber and additionally it also has low insertion loss.

- $\quad$ Electronic Dispersion Compensation (EDC):

Electronic compensation technique makes use of electronics in conjunction with optics so as to compensate dispersion. There are some ways during which EDC is used to compensate dispersion. One in all them is compensating initial order PMD within the fiber by cancelling it out with a complementary PMD vector made at the receiver finish. Dispersion compensation by use of equalization circuits is that the commonest technique used to compensate dispersion by EDC.[2]

\section{- Digital Filters:}

This is a new idea of using all Pass Optical Filter the most effective means of dispersion compensation using digital filters. Optical all pass filters are lossless filters that are capable of tuning a desired section response by manipulating the amount of stages keeping magnitude response of the system constant [2].

B. Dispersion in Fibers

\section{- $\quad$ Chromatic Dispersion:}

Chromatic or intramodal dispersion might occur all told varieties of optical fiber and results from the finite spectral line width of the optical source. Since optical sources don't emit simply one frequency however a band of frequencies (in the case of the injection laser like only a fraction of a percent of the middle frequency, whereas for the light-emitting diode it's probably to be a significant percentage), then there is also propagation delay variations between the various spectral parts of the transmitted signal. This cause broadening of every transmitted mode and therefore intramodal dispersion. The delay variations could also caused by the dispersive properties of the wave guide material (material dispersion) and also guidance effects at intervals the fiber structure (waveguide dispersion).

Material Dispersion:

Pulse broadening because of material dispersion results from the various group velocities of the varied spectral parts launched into the fiber from the optical supply. It happens once the phase velocity of a plane wave propagating within the dielectric medium varies nonlinearly with wavelength, and a material is alleged to exhibit material dispersion once the second differential of the refractive index with respect to wavelength isn't zero. 
Waveguide Dispersion:

The wave guiding of the fiber can also produce chromatic dispersion. This results from the variation in group speed with wavelength for a specific mode. Considering the ray theory approach, it's like the angle between the ray and therefore the fiber axis variable with wavelength that subsequently results in a variation within the transmission times for the rays, and therefore dispersion. For one mode whose propagation constant is $\beta$, the fiber exhibits waveguide dispersion once $\mathrm{d} 2(\beta) / \mathrm{d} 2(\lambda) \neq 0$.

\section{- Intermodal Dispersion:}

Pulse broadening due to intermodal dispersion (sometimes named simply as modal or mode dispersion) results from the propagation delay variations between modes inside a multimode fiber. Because the totally different modes that represent a pulse during a multimode fiber travel along the channel at different group velocities, the pulse width at the output relies upon the transmission times of the slowest and fastest modes. Therefore multimode step index fibers exhibit a large quantity of intermodal dispersion which supplies the best pulse broadening. However, intermodal dispersion in multimode fibers is also reduced by adoption of an optimum ratio profile that is provided by the nearparabolic profile of most graded index fibers.

\section{- $\quad$ Polarization Mode Dispersion:}

Polarization mode dispersion is another supply of limitation that happens because, though the single-mode fiber will sustain only 1 transverse mode, it will carry this mode with 2 completely different polarizations, and slight imperfections or distortions during a fiber will alter the propagation velocities for the 2 polarizations [5]. This development is termed birefringence. The dispersion is proportional to the length of the fiber. If the length is increased the width becomes bulk and also the magnitude reduces [6].

\section{CONCLUSION}

This paper has reviewed the in the main analysis trends and proposed dispersion compensation using Fiber Bragg Grating (FBG) in Optical Communication. This paper studied is Dispersion Compensation Techniques in optical fiber communication system and dispersion in fiber. We tend to additionally study the various techniques used to compensate the chromatic dispersion and the dispersion compensation using Fiber Bragg Grating at different fiber lengths and at different FBG.

\section{REFERENCES}

[1] Kumar, Kaushal, et al. "Performance Analysis of dispersion compensation using Fiber Bragg Grating (FBG) in Optical Communication." International Journal of Current Engineering and Technology 4.3 (2014): 1527-1531..

[2] Mr.TusharKantPanda, ,Dr.Krishra Chandra patra and Dr.Nalinikant Barapanda, "Enhancing Transmission Performance of Fiber Optic Link Using Fiber Bragg Grating For Effective Dispersion Compensation”, International Journal of Computer Engineering and Applications, Volume X, Issue VII, July 16 www.ijcea.com ISSN 2321-3469.

[3] Bibhu Prasad, Dr. K. C. Patra, Dr. N.K Barpanda "Performance Analysis of Fiber Optical Communication using Fiber Bragg Grating as Dispersion Compensator" IJAREEIE Vol. 5, Issue 4, April 2016.

[4] Anand, Sameer, P. K. Raghav, and Divya Kumar. "Analysis on dispersion compensation using Post FBG with EDFA." International J ournal of Scientific \& Engineering Research 4.9 (2013): 1809-1813.

[5] Ranjana Rao, Dr. Suresh Kumar "Performance Analysis of Dispersion Compensation using FBG and DCF in WDM Systems: A Review", International Journal of Electronics, Electrical and Computational System IJEECS ISSN 2348-117X Volume 5, Issue 10 October 2016.

[6] Parida, B. Prasad1 B. Mallick2 AK. "Fiber Bragg Grating as a Dispersion Compensator in an Optical Transmission System Using Optisystem Software." (2015).

[7] R. Udayakumar, V. Khanaa and T. Saravanan "Chromatic Dispersion Compensation in Optical Fiber Communication System and its Simulation”, Indian Journal of Science and Technology 6.6 (2013).

[8] S. O. Mohammadi1, Saeed Mozaffaril and M. Mahdi Shahidi2 "Simulation of a transmission system to compensate dispersion in an optical fiber by chirp gratings “. International Journal of the Physical Sciences Vol. 6(32), pp. 7354 - 7360, 2 December, 2011.

[9] Huang Liqun, Song Xin, Liu Fulai, Shen Li, and Han Laiquan "Computer Simulation of 40Gb/s Optical Fiber Transmission Systems with a Fiber Grating Dispersion Compensator "20IO International Conference On Computer Design And Appliations (ICCDA 2010)

[10] S Sujith and K G Gopchandran "A Simulation study on DCF compensated SMF using OptSim". 2010 International congress on Ultra Modern Telecommunications and Control Systems and Workshops (ICUMT) 978-1-4244-7286-4/10/\$26.00 C2010 IEEE Department of Optoelectronics, University of Kerala,Thiruvananthapuram - 695 581, India)

[11] Gnanam Gnanagurunathan, Faidz Abd. Rahman "Comparing FBG and DCF as dispersion compensators in the long haul narrowband WDM systems".

[12] Isabelle Riant, Salim Gurib, Josselyne Gourhant, Pierre Sansonetti, Christian Bungarzeanu, and Raman Kashyap "Chirped Fiber Bragg Gratings for WDM Chromatic Dispersion Compensation in Multispan 10-Gb/s Transmission" IEEE journal of selected topics in quantum electronics, vol. 5, no. 5, september/October 1999. 